\title{
BMJ Open Delayed puberty in boys in central Sweden: an observational study on diagnosing and management in clinical practice
}

\author{
Maria Rodanaki (D , , Eva Rask, $^{2,3}$ Maria Lodefalk ${ }^{1,3}$
}

To cite: Rodanaki M, Rask E, Lodefalk M. Delayed puberty in boys in central Sweden: an observational study on diagnosing and management in clinical practice. BMJ Open 2022;12:e057088. doi:10.1136/ bmjopen-2021-057088

- Prepublication history and additional supplemental material for this paper are available online. To view these files, please visit the journal online (http://dx.doi.org/10.1136/ bmjopen-2021-057088).

Received 08 September 2021 Accepted 11 January 2022

Check for updates

(c) Author(s) (or their employer(s)) 2022. Re-use permitted under CC BY-NC. No commercial re-use. See rights and permissions. Published by BMJ.

${ }^{1}$ Department of Pediatrics and Adolescent Medicine, Faculty of Medicine and Health, Örebro University, Örebro, Sweden ${ }^{2}$ Department of Medicine, Faculty of Medicine and Health, Örebro University, Örebro,

Sweden

${ }^{3}$ University Health Care Centre, Faculty of Medicine and Health, Örebro University, Örebro, Sweden

Correspondence to Dr Maria Rodanaki; maria.rodanaki@oru.se

\section{ABSTRACT}

Objectives To compare the usefulness of the classical definition of delayed puberty (DP) in boys with puberty nomograms and to describe the management of DP in boys in a hospital-based setting.

Study design Observational retrospective multicentre study with a short-term follow-up.

Setting and participants Boys diagnosed with DP during 2013-2015 at paediatric departments in four counties in central Sweden. The medical records of 165 boys were reviewed.

Primary and secondary outcome measures Number of boys with DP after re-evaluation of the diagnosis according to the classical definition in comparison with puberty nomograms. Description of investigations performed and treatment provided to boys with DP.

Results In total, 45 and 58 boys were found to have DP according to the classical definition and the nomograms, respectively. Biochemical and/or radiological testing was performed in $91 \%$ of the 58 boys, but an underlying disease was only found in $9 \%$ of them. Approximately $79 \%$ of the boys received testosterone treatment, either as injections of testosterone enanthate or as testosterone undecanoate.

Conclusions Puberty nomograms may be helpful instruments when diagnosing pubertal disorders in boys as they are not limited to an age close to 14 years and also identify boys with pubertal arrest. The majority of boys with DP undergo biochemical or radiological examinations, but underlying diseases are unusual emphasising the need for structural clinical practice guidelines for this patient group.

\section{INTRODUCTION}

Central puberty starts when the hypothalamicpituitary-gonadal axis has been activated. The first clinical sign of puberty in boys is testicular enlargement. ${ }^{1}$ The exact mechanisms regulating the onset of puberty are not fully understood, but genetic, metabolic and lifestyle factors appear to be involved. ${ }^{23}$

Delayed puberty (DP) has classically been defined as lack of pubertal signs at the age of 14 years in boys. In addition to the classical definition of DP, the use of puberty
Strengths and limitations of this study

- The re-evaluation of the diagnosis by a thorough review of medical records in a hospital-based, geographically well-defined population including both secondary and tertiary paediatric centres.

- The highly accessible medical service of the Swedish healthcare system increasing the opportunity to include a broad sample of boys with delayed puberty.

- The small study population and the retrospective design were limitations of the study.

nomograms has been suggested for the improvement of the diagnostic process, as they can also identify slow pubertal progression. ${ }^{4}$ Puberty nomograms have been introduced in the Netherlands ${ }^{56}$ and are in use in the UK. ${ }^{7}$ Furthermore, they have been used for the evaluation of DP in Danish boys ${ }^{4}$ and for the description of pubertal progress in Danish girls. ${ }^{8}$ However, as far as we know, no previous study has evaluated puberty nomograms in a Swedish setting.

DP is much more common in boys than in girls. ${ }^{9}$ The most common cause of DP in boys is constitutional delay of growth and puberty (CDGP), which is a self-limiting condition characterised by short stature at the time of diagnosis and a positive family history of DP. ${ }^{9-12}$ However, persistent or functional hypogonadism needs to be excluded, leading to a variable use of biochemical and radiological investigations. The diagnostic approach to boys with DP has been reported to vary substantially between practitioners, ${ }^{10}$ indicating a need for a structured evaluation of the clinical management of these patients. Furthermore, previous descriptions of boys with DP have often been limited to tertiary paediatric centres, ${ }^{411}$ which may result in over-reporting of the most severe cases.

DP in boys can be treated with testosterone. ${ }^{13}$ A short treatment period with 
low-dose testosterone usually initiates pubertal onset in boys with CDGP. Intramuscular injections of testosterone every month are most often used, but transdermal testosterone patches, gels and oral testosterone have also been proposed. ${ }^{14}$

The aims of the present study were to compare the usefulness of the classical definition with that of puberty nomograms in diagnosing DP in Swedish boys and to describe the clinical management of these patients, in terms of investigations performed and treatment provided in a geographically well-defined, hospital-based population including both secondary and tertiary paediatric centres.

\section{PATIENTS AND METHODS \\ Participants}

Every paediatric department in four counties in central Sweden (Örebro, Södermanland, Västmanland and Dalarna) was included in the study. These departments provide secondary and tertiary medical care for all adolescents with a suspected pubertal disorder in the area. The total population in this area was 1108024 inhabitants in 2014. All outpatient visits at these departments are routinely registered with patient's International Statistical Classification of Diseases and Related Health Problems (ICD) code. These registers were run through for all boys diagnosed with DP (ICD-10 code E30.0) from 1 January 2013 to 31 December 2015. To ensure that all boys with DP were identified, patients with diagnoses potentially related to DP were also included in the search by the use of the following ICD-10 codes: E30, E30.8 and E30.9 (disorders of pubertal development); E22.1 (hyperprolactinaemia); E23.0, E23.0E and E23.0F (hypopituitarism); E29.1, E29.8 and E29.9 (testicular dysfunction); and E34.3 (short stature due to endocrine disorder).

Identified patients were given written information on the study, and consent for the review of their medical record was requested. After the approval of the second application to the Regional Board of Ethics, it was possible to include all patients (even when written consent was missing) at the Department of Paediatrics, Örebro University Hospital (the main centre), except for those who had actively declined participation.

\section{Patient and public involvement}

There was no patient or public involvement in the design of the study nor in the interpretation of findings.

\section{Procedures and definitions}

The medical records were reviewed using a data capture form (see online supplemental material) to verify the diagnosis of DP using the classical definition: testicular volume $<4 \mathrm{~mL}$ by the age of $\geq 14$ years. ${ }^{1}$ However, many boys did not attend the paediatric outpatient clinic at exactly 14 years of age and many boys had entered puberty at the time of the visit. Therefore, the accuracy of the diagnosis based on the classical definition was evaluated by combining pubertal stage and age at the time of the visit while keeping in mind that puberty usually progresses by one Tanner stage per year. ${ }^{15}$ After this structural evaluation, each diagnosis was labelled as certain, probable, inaccurate or unclear as shown in online supplemental table S1. For example, when a 14.5 -year-old boy had a testicular volume of $5 \mathrm{~mL}$ and was in Tanner stage 2, the label 'probable' DP was used. The puberty nomogram used for comparison with the classical definition was developed in Denmark. ${ }^{4}$ In agreement with its instruction, a boy with a genital stage or testicular volume $>2$ SD below the mean for his age was considered to have DP.

Data on clinical characteristics, investigations performed, treatment provided and short-term outcome of the treatment were collected from the medical records. Body weight and height were transformed into SD scores using Swedish reference growth data. ${ }^{16}$

\section{Laboratory and radiological analyses}

Investigations were performed at the discretion of each paediatrician. Blood and urine samples were analysed at each hospital's Department of Laboratory Medicine except for karyotype analysis, which was performed at the Department of Clinical Genetics, Uppsala University Hospital, Uppsala, Sweden. Radiological investigations were performed at each hospital's radiological department. The Greulich and Pyle method ${ }^{17}$ was used to describe bone age.

\section{Statistics}

Data are presented as absolute numbers (per cent), the mean (SD) or median (min-max), as appropriate. The $\chi^{2}$ test, Student's t-test and the Mann-Whitney U test were used, as appropriate. Statistical significance was set at $\mathrm{p}<0.05$ for two-sided tests. Calculations were performed in IBM SPSS Statistics for Windows V.24 (IBM).

\section{RESULTS}

\section{Study population}

The search identified 215 boys with the diagnosis of DP (ICD-10 code: E30.0), of whom $42 \%$ consented to participate. After the approval of the second application to the Regional Board of Ethics, the inclusion rate in Örebro county (the main centre) increased to $83 \%$. The search also identified 186 boys with other ICD-10 codes, and 74 of them could be included in the study, but after the review on their medical records, none of them were found to have DP. In total, the medical records of 165 boys were reviewed, and of them, 91 were diagnosed with DP in the records. After the re-evaluation of the diagnosis based on the classical definition, 30 boys were considered as certain DP cases and 15 as probable cases. After the nomogram evaluation, 58 boys were found to have DP or slow pubertal progression (figure 1). Boys with DP or slow pubertal progression according to the nomogram living in the counties of Södermanland, Västmanland or Dalarna (where the inclusion rate was low) did not differ 


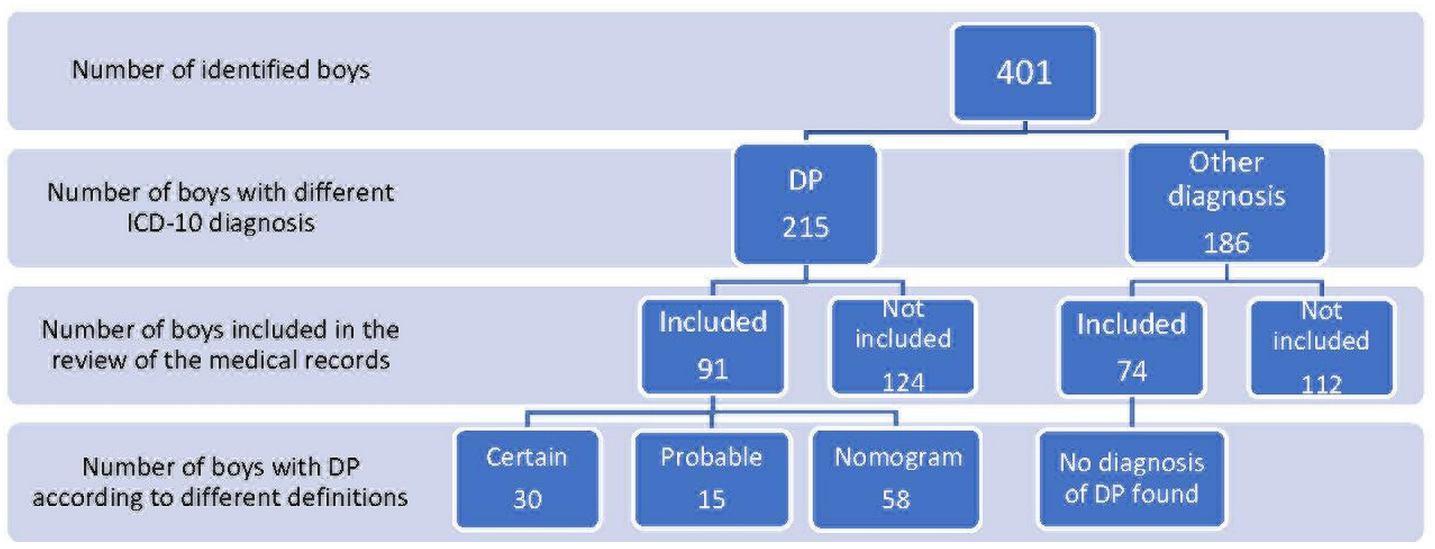

Figure 1 Flow chart of the study population. DP, delayed puberty; ICD-10, International Statistical Classification of Diseases and Related Health Problems version 10.

significantly in clinical characteristics from boys living in Orebro county (where the inclusion rate was sufficient). Therefore, all boys with DP according to the nomograms $(n=58)$ were analysed as one group regarding investigations performed and treatment provided.

\section{Comparison of the classical definition and puberty nomograms}

Based on the classical definition, 45 of the 91 boys (49\%) diagnosed with DP in the clinical setting were found to have certain or probable DP and the corresponding number based on nomogram evaluation was $64 \%$. The majority of boys (42 of 45 , ie, $93 \%$ ) identified as having DP according to the classical definition were also classified as having DP by the use of the nomograms. However, only $72 \%$ of the boys defined as having DP or slow pubertal progression by the nomograms were identified as having DP by the classical definition (42 of 58 boys, table 1 ).

\section{Clinical characteristics, investigations performed, treatment provided and short-term follow-up}

Short stature according to the pubertal growth chart was found in 39 of the 58 boys (67\%) with DP according to the nomograms. The majority of the boys $(86 \%)$ had CDGP. Of the 37 patients with information available in the medical record on psychosocial distress, $65 \%$ reported such a complication, including feelings of sadness, social withdrawal, giving up sport activities and bullying. Most of the boys had another medical problem prior to the pubertal delay, such as asthma or allergies $(n=10)$, attention deficit and hyperactivity disorder (ADHD) $(n=7)$, or

\begin{tabular}{|c|c|c|}
\hline \multirow{2}{*}{$\begin{array}{l}\text { Delayed puberty } \\
\text { according to classical } \\
\text { definition }\end{array}$} & \multicolumn{2}{|c|}{$\begin{array}{l}\text { Delayed puberty according to } \\
\text { puberty nomograms }\end{array}$} \\
\hline & Yes & No \\
\hline Yes & 42 & 3 \\
\hline No & 16 & 30 \\
\hline
\end{tabular}

epilepsy or another neurological disease, including intellectual disability $(\mathrm{n}=7)$ (table 2$)$.

Biochemical testing and/or radiological assessment was performed in $91 \%$ of the boys. Serum levels of insulinlike growth factor 1 were lower than expected in nine of 20 tested boys. Bone age assessment was performed in 12 boys and all of them had delayed bone age (mean delay: 26 months $( \pm 11)$ ). Karyotype testing was performed in seven boys; all had normal results. A gonadotropin-releasing hormone stimulation test was performed in three boys, growth hormone testing was performed in four boys and human chorionic gonadotropin testing was performed in one boy. MRI of the brain and the pituitary gland was performed in seven boys. The following diagnoses were found: Kallmann syndrome $(n=2)$, congenital pituitary malformation $(\mathrm{n}=1)$, hypergonadotropic hypogonadism $(\mathrm{n}=1)$ and coeliac disease $(\mathrm{n}=1)$.

Testosterone treatment was prescribed to 45 boys. An intramuscular injection of testosterone enanthate (75

Table 2 Clinical characteristics of boys with delayed puberty in central Sweden

\begin{tabular}{ll}
\hline Age (years) $(\mathrm{n}=58)$ & $15.2(0.83)$ \\
\hline Weight SDS $(\mathrm{n}=54)$ & $-1.42(1.81)$ \\
\hline Height SDS $(\mathrm{n}=56)$ & $-2.31(1.23)$ \\
$\begin{array}{l}\text { Referred from the school healthcare system } \\
(\mathrm{n}=43)\end{array}$ & $37 \%$ \\
$\begin{array}{l}\text { Positive family history of delayed puberty } \\
(\mathrm{n}=40)\end{array}$ & $85 \%$ \\
$\begin{array}{l}\text { Any comorbidity }(\mathrm{n}=58) \\
\text { Any reported psychosocial distress }(\mathrm{n}=37)\end{array}$ & $65 \%$ \\
\hline $\begin{array}{l}\text { Diagnosed with CDGP }(\mathrm{n}=57) \\
\text { Treated with testosterone }(\mathrm{n}=57)\end{array}$ & $86 \%$ \\
\hline
\end{tabular}

Values are mean (SD) or per cent. Height SDS was based on the pubertal growth spurt and not the prepubertal growth rate. $\mathrm{n}$ is the number of patients with information on that variable available in the medical record.

CDGP, constitutional delay of growth and puberty; SDS, SD score. 
$\mathrm{mg}$ ) was given monthly to 33 of them $(73 \%)$. The median duration of the treatment was 6 months (4-12 months). Patients with permanent gonadotropin deficiency received prolonged treatment. Twelve boys were given two intramuscular injections of testosterone undecanoate separated by an interval of 12 weeks (the most commonly used dose was $200 \mathrm{mg}$ per injection). Some of these 12 patients required higher doses or prolonged therapy.

The testosterone treatment was successful (ie, a higher Tanner stage or the patient was satisfied with the outcome) in $80 \%$ of the treated boys regardless of type of testosterone given. Both types of treatment regimens were well tolerated in $89 \%$ of the patients. Reported potential adverse events were few but included a sickle cell crisis and priapism in a patient with sickle cell anaemia, and reactive arthritis or aphthous stomatitis in one patient each.

\section{DISCUSSION}

In this hospital-based observational study of a geographically well-defined population including both secondary and tertiary paediatric centres, we found a high concordance between the classical definition and puberty nomograms in boys with DP even though the nomograms identified more patients. Short stature and psychosocial distress were common complaints. A high proportion of the boys (91\%) underwent some type of biochemical or radiological investigation, but investigations performed varied widely, and in only approximately $9 \%$ of the patients an aetiological disorder was found. The diagnosis of DP could only be verified in approximately half of the cases after the structured re-evaluation of data. Similar low verification rates have been presented before in a Danish ${ }^{4}$ and an American study. ${ }^{18}$ In the latter, only $71 \%$ of the practitioners used the classical cut-off age for defining DP. The low verification rate found in the present study may be due to the variable ages of the patients at the time of the visit to the paediatrician, making it difficult to use the classical definition, and the strict criteria used in the study when re-evaluating the diagnosis. In the clinical situation, paediatricians may be less strict taking into account a combination of clinical data, biochemical test results and the psychosocial burden of the patients when diagnosing the condition. ${ }^{18}$

The superiority of puberty nomograms to the classical definition found in the present study was probably due to the fact that nomograms are not restricted to an age close to 14 years and many of the patients reviewed in this study ( $n=61$ ) did not see their doctor at 14 years of age or they were not completely prepubertal making it hard to diagnose them with DP by a strict use of the classical definition. Puberty nomograms also identify adolescents with slowly progressing puberty and, consequently, they would identify more patients with pubertal disorders, as shown in both the Danish study ${ }^{4}$ and the present study. Patients with diagnoses other than CDGP, for example, Klinefelter syndrome, may have a normal pubertal onset but an abnormal pubertal progression, and for this subgroup, puberty nomograms are useful for monitoring the pubertal progression. ${ }^{19}$ However, most boys with signs of late puberty have CDGP and if they are assessed at an older age than 14 years and then have some pubertal signs, we propose that puberty nomograms are helpful in diagnosing DP, as well as monitoring their pubertal progression.

The clinical characteristics found in the present study are in good agreement with previous findings. ${ }^{49}$ However, the proportion of boys diagnosed with CDGP in this study $(86 \%)$ was higher than that in other studies $(63 \%-82 \%),{ }^{4-11}$ which may be due to differences in study populations where our study did include tertiary and secondary centres and due to differences in accessibility to health services between countries. More than half of the patients in our study had some comorbidities, possibly partly explained by the study design, including patients from paediatric departments only. Older ${ }^{9}$ as well as more recent studies ${ }^{20}$ have described a possible association between DP and ADHD, which could also be seen in our study, where $12 \%$ of the boys with DP also had ADHD. This prevalence is higher than that described globally, as well as in Sweden $(5 \%-7 \%) .{ }^{20}{ }^{21}$ It has been speculated that genetic factors, hormonal effects, ADHD medication side effects or increased awareness of growth deviations may be possible explanations of this association, but more studies are needed for a deeper understanding of the observation. $^{920}$

Although psychosocial distress has previously been mentioned as a common feature of DP in boys, ${ }^{422} 23$ the types of distress and their frequencies are not completely known. In the present study, a considerable proportion $(65 \%)$ of the boys with DP reported psychosocial distress, indicating a need for high awareness of this complication in this patient group.

The large proportion of boys investigated with blood samples, stimulation tests or radiological examinations in the present study underlines that a more structured diagnostic approach is needed to avoid unnecessary testing and to optimise the cost-effectiveness as suggested previously. ${ }^{12}$ This is especially important considering the fact that the majority of boys with DP have CDGP, which is an extreme variant of the normal spectrum of pubertal development. No Swedish guidelines on the management of DP existed during the study time period, which possibly can explain some of the variation seen in the management of the condition in the present study. However, this variation has also been shown in previous studies from other countries. $^{18}$

The traditional treatment for DP in boys is monthly intramuscular injections of testosterone enanthate. ${ }^{14}$ In the present study, most boys were given this treatment regimen, but some boys were given intramuscular injections of testosterone undecanoate, which is approved for adult men with hypogonadism ${ }^{24-26}$ and for pubertal induction in adult men. ${ }^{24}$ In addition, testosterone undecanoate has been used orally in prepubertal boys, ${ }^{4}$ but 
to the best of our knowledge, this is the first report on intramuscular injections of testosterone undecanoate in prepubertal boys. The administration of testosterone undecanoate has the advantage of a longer injection time interval. This treatment regimen seemed to be well tolerated and the short-term outcome seemed to be favourable, but these findings should be interpreted with caution since only very few patients received this treatment and because of the design of the present study. Future randomised controlled intervention studies are needed to appropriately investigate which treatment regimen is most suitable in boys with DP.

Strengths of the present study are the Swedish healthcare system including regular medical check-ups of growth and development of all children and adolescents at school healthcare units and the low economic cost for the patient, making medical advice highly accessible and thus increasing the possibility to include a broad sample of boys with DP in the study; the thorough review of the medical records to validate the diagnoses; and the welldefined geographical population including patients from both secondary and tertiary centres. One of the limitations of the present study is the small sample size, which was due to the low response rate, and it may affect the implication of our findings. The retrospective design of the study is another limitation, as well as data collection from medical records only. Additionally, the inclusion of several centres and practitioners may explain some of the variation found in clinical management. On the other hand, the multicentre design may increase the generalisability of our findings. Despite the inclusion of both secondary and tertiary centres, some boys with DP may not be aware of the diagnosis or may not be disturbed by the late development and consequently may not seek medical advice introducing a risk of selection bias in the present study.

In conclusion, puberty nomograms identify more boys with pubertal disorders compared with the classical definition of DP since they are not limited to an age close to 14 years or to late pubertal onset. The majority of boys with DP undergo biochemical or radiological examinations, but underlying diseases are seldom detected indicating a need for improved clinical guidelines for this patient group in order to reduce the number of unnecessary investigations.

Acknowledgements We thank the paediatricians Anders Andersson, C-G Arvidsson, Charlotte Nylander and Helena Demetriades for help with the inclusion of patients.

Contributors All authors (MR, ML and ER) contributed to the design of the study. MR collected the data, performed the statistical analysis and wrote the first draft of the manuscript with support from ML and ER. All authors (MR, ML and ER) were involved in revising the manuscript. MR is responsible for the overall content (guarantor).

Funding This study received financial support from the Research Committee and ALF funding, Region Örebro County.

Competing interests None declared.

Patient consent for publication Not required.
Ethics approval This study involves human participants and was approved by the Regional Board of Ethics in Uppsala, Sweden (2016/296). Due to low response rate, an additional application was sent and approved (2016/296/1). Participants gave informed consent to participate in the study before taking part.

Provenance and peer review Not commissioned; externally peer reviewed.

Data availability statement Data are available upon reasonable request. Data are available by emailing maria.rodanaki@oru.se.

Supplemental material This content has been supplied by the author(s). It has not been vetted by BMJ Publishing Group Limited (BMJ) and may not have been peer-reviewed. Any opinions or recommendations discussed are solely those of the author(s) and are not endorsed by BMJ. BMJ disclaims all liability and responsibility arising from any reliance placed on the content. Where the content includes any translated material, BMJ does not warrant the accuracy and reliability of the translations (including but not limited to local regulations, clinical guidelines, terminology, drug names and drug dosages), and is not responsible for any error and/or omissions arising from translation and adaptation or otherwise.

Open access This is an open access article distributed in accordance with the Creative Commons Attribution Non Commercial (CC BY-NC 4.0) license, which permits others to distribute, remix, adapt, build upon this work non-commercially, and license their derivative works on different terms, provided the original work is properly cited, appropriate credit is given, any changes made indicated, and the use is non-commercial. See: http://creativecommons.org/licenses/by-nc/4.0/.

ORCID iD

Maria Rodanaki http://orcid.org/0000-0003-2641-5629

\section{REFERENCES}

1 Marshall WA, Tanner JM. Variations in the pattern of pubertal changes in boys. Arch Dis Child 1970;45:13-23.

2 Palmert MR, Boepple PA. Variation in the timing of puberty: clinical spectrum and genetic investigation. $J$ Clin Endocrinol Metab 2001;86:2364-8.

3 Alotaibi MF. Physiology of puberty in boys and girls and pathological disorders affecting its onset. $J$ Adolesc 2019;71:63-71.

4 Lawaetz JG, Hagen CP, Mieritz MG, et al. Evaluation of 451 Danish boys with delayed puberty: diagnostic use of a new puberty nomogram and effects of oral testosterone therapy. J Clin Endocrinol Metab 2015;100:1376-85.

5 van Buuren S, Ooms JCL. Stage line diagram: an age-conditional reference diagram for tracking development. Stat Med 2009;28:1569-79.

6 van Buuren S. Growth charts of human development. Stat Methods Med Res 2014;23:346-68.

7 Boys childhood and puberty close monitoring growth chart RCPCH2013. Available: https://www.rcpch.ac.uk/sites/default/files/ Boys_childhood_and_puberty_close_monitoring_growth_chart.pdf

8 Lindhardt Johansen M, Hagen CP, Mieritz MG, et al. Pubertal progression and reproductive hormones in healthy girls with transient Thelarche. J Clin Endocrinol Metab 2017;102:1001-8.

9 Sedlmeyer IL, Palmert MR. Delayed puberty: analysis of a large case series from an academic center. J Clin Endocrinol Metab 2002;87:1613-20.

10 Abitbol L, Zborovski S, Palmert MR. Evaluation of delayed puberty: what diagnostic tests should be performed in the seemingly otherwise well adolescent? Arch Dis Child 2016;101:767-71.

11 Varimo T, Miettinen PJ, Känsäkoski J, et al. Congenital hypogonadotropic hypogonadism, functional hypogonadotropism or constitutional delay of growth and puberty? an analysis of a large patient series from a single tertiary center. Hum Reprod 2017;32:147-53.

12 Sedlmeyer IL, Hirschhorn JN, Palmert MR. Pedigree analysis of constitutional delay of growth and maturation: determination of familial aggregation and inheritance patterns. $J$ Clin Endocrinol Metab 2002;87:5581-6.

13 Stancampiano MR, Lucas-Herald AK, Russo G, et al. Testosterone therapy in adolescent boys: the need for a structured approach. Horm Res Paediatr 2019;92:215-28.

14 Wei C, Crowne EC. Recent advances in the understanding and management of delayed puberty. Arch Dis Child 2016;101:481-8.

15 Roche AF, Wellens R, Attie KM, et al. The timing of sexual maturation in a group of US white youths. J Pediatr Endocrinol Metab 1995;8:11-18.

16 Wikland KA, Luo ZC, Niklasson A, et al. Swedish population-based longitudinal reference values from birth to 18 years of age for height, weight and head circumference. Acta Paediatr 2002;91:739-54. 
17 Greulich WW, Pyle SI. Radiographic atlas of skeletal development of the hand and wrist. Am J Med Sci 1959;238:393.

18 Zhu J, Feldman HA, Eugster EA, et al. Practice variation in the management of girls and boys with delayed puberty. Endocr Pract 2020;26:267-84.

19 Tanner M, Miettinen PJ, Hero M, et al. Onset and progression of puberty in Klinefelter syndrome. Clin Endocrinol 2021. doi:10.1111/ cen.14588. [Epub ahead of print: 14 Sep 2021].

20 Ohlsson Gotby V, Söder O, Frisén L, et al. Hypogonadotrophic hypogonadism, delayed puberty and risk for neurodevelopmental disorders. J Neuroendocrinol 2019;31:e12803.

21 Sayal K, Prasad V, Daley D, et al. Adhd in children and young people: prevalence, care pathways, and service provision. Lancet Psychiatry 2018;5:175-86.
22 Maggi M, Buvat J. Standard operating procedures: pubertas tarda/ delayed puberty--male. J Sex Med 2013;10:285-93.

23 Dwyer AA. Psychosexual effects resulting from delayed, incomplete, or absent puberty. Curr Opin Endocr Metab Res 2020;14:15-21.

24 Santhakumar A, Miller M, Quinton R. Pubertal induction in adult males with isolated hypogonadotropic hypogonadism using longacting intramuscular testosterone undecanoate 1-g depot (Nebido). Clin Endocrinol 2014;80:155-7.

25 Giagulli VA, Triggiani V, Carbone MD, et al. The role of long-acting parenteral testosterone undecanoate compound in the induction of secondary sexual characteristics in males with hypogonadotropic hypogonadism. J Sex Med 2011;8:3471-8.

26 Wolf $\mathrm{J}$, Keipert $\mathrm{D}$, Motazedi $\mathrm{H}$, et al. Effectiveness and tolerability of parenteral testosterone undecanoate: a post-marketing surveillance study. Aging Male 2017;20:225-34. 